Article

\title{
Layer-by-Layer Deposition of Hyaluronan and Quercetin-Loaded Chitosan Nanoparticles onto Titanium for Improving Blood Compatibility
}

\author{
Xingda $\mathrm{Wu}^{1,+}$, Cuijuan Liu ${ }^{1,+}$, Hongpeng Chen ${ }^{1}$, Yanfang Zhang ${ }^{1}$, Lin Li $^{2}$ and Nan Tang ${ }^{1, *(D)}$ \\ 1 School of Biomedical Engineering, School of Pharmacy, Guangdong Medical University, Dongguan 523808, \\ China; shuradio@shu.edu.cn (X.W.); liu-cj@foxmail.com (C.L.); chenhpgdmc@foxmail.com (H.C.); \\ zjzyf2006@163.com (Y.Z.) \\ 2 Guangdong Provincial Key Laboratory of New Drug Screening, Guangzhou Key Laboratory of Drug \\ Research for Emerging Virus Prevention and Treatment, School of Pharmaceutical Sciences, \\ Southern Medical University, Guangzhou 510515, China; li75lin@126.com \\ * Correspondence: tn6559@foxmail.com \\ + These authors contributed equally to this work.
}

Received: 23 January 2020; Accepted: 29 February 2020; Published: 9 March 2020

\begin{abstract}
Surface modification is an effective way to improve the hemocompatibility of biomaterials. Quercetin has significant anticoagulation and antithrombotic effects, and thus it is a promising candidate agent for the surface modification of blood-contacting materials. In this study, quercetin was successfully encapsulated in tripolyphosphate-chitosan nanoparticles (TCs) based on the ionic gelation of chitosan with tripolyphosphate (TPP) anions. Then, hyaluronan acid (HA)/quercetin-loaded TPP-chitosan nanoparticle (QTCs) films, in addition to HA/TCs films, were prepared separately using an electrostatic layer-by-layer self-assembly technique. The encapsulation of quercetin in the chitosan nanoparticles was confirmed by UV spectra. The quercetin-loaded multilayer coatings were also successfully self-assembled, as confirmed by the UV spectra and contact angle measurements. Platelet adhesion experiments were carried out with platelet-enriched plasma so as to evaluate the blood compatibility of the different samples. There were many platelets on the surfaces of the glass and HA/TC-coated titanium, which were partially activated but not aggregated. Meanwhile, many more platelets were observed on the uncoated titanium surfaces, most of which developed pseudopodia. By contrast, the platelet adhesion and activation were reduced remarkably on the surface of the HA/QTC-coated titanium. These results showed that the multilayer coatings containing quercetin could act as potential biomaterials to improve the anticoagulation performance of blood-contacting materials.
\end{abstract}

Keywords: biomaterial; surface modification; self-assembly; multilayer; blood compatibility

\section{Introduction}

In the design of blood-contacting implants, such as artificial blood vessels and vascular stents, there are two major issues that should be considered, namely, antithrombosis and endothelialization [1]. Native endothelium plays a unique role in maintaining vascular homeostasis, which includes active antithrombosis, inhibiting the proliferation of smooth muscle cells, and reducing intimal hyperplasia [2,3]. Thus, the endothelialization of implant surfaces may provide fully blood-compatible interfaces. However, we know that it takes some time to complete the endothelialization of implant surfaces in vivo. Therefore, the blood compatibility of an implant is the first consideration, especially in the initial implantation. 
Many approaches have been explored to improve the blood compatibility of blood-contacting medical implants. Surface modification enables the combination of a material's bulk properties with the desired biological attributes, and it has become the most popular method for preventing blood clotting. Various anticoagulant molecules, including heparin [4,5], citric acid [6], hirudin [7], and some enzymes [8], have been used to develop clinical devices with anticoagulant properties. In addition, endothelial cell seeding has also been used in surface coatings [9]. Biomaterials functionalized with endothelial cell seeding may function in a similar way as the endothelial surface itself, but the poor adhesion and slow formation process limit its clinical application [10]. To date, there are no blood-contacting materials used in clinical settings that can meet all of the hemocompatibility requirements, whether short-term applied or long-term implanted [11]. Thus, the field of biomaterials modification, from the perspective of improving the blood compatibility, is still evolving. More innovative approaches and strategies need to be explored and developed.

The layer-by-layer (LbL) self-assembly technique is a simple and facile approach for the immobilization of biomolecules onto various surfaces, and it has already been extensively applied in the surface modification of biomaterials. Compared with the classic chemical immobilization, the LbL technique has the lowest demand for reactive chemical bonds and efficiently keeps the molecular activity. The LbL self-assembled multilayers produce a more stable coating than that prepared by physical adsorption because of the electrostatic attractions between the different layers. In many previous studies, the LbL technique was used to immobilize heparin onto various kinds of biomaterials. As an important anticoagulant in clinics, heparin was the most popular surface modifier. Li et al. constructed LbL-assembled heparin/fibronectin multilayers on titanium implants, and these multilayers displayed excellent antithrombotic properties [12]. Similarly, Su et al. developed a dual drug-eluting stent, with Duraflo heparin and sirolimus coated layer-by-layer onto a Multi-Link PIXEL stent, which showed prolonged resistance to thrombosis [13]. Chou et al. demonstrated that the anticoagulation performance of collagen/heparin multilayers on titanium surfaces was superior to that of an uncoated one [14]. Meng et al. designed a chitosan/heparin LbL-coated coronary stent and tested both in in vitro and in vivo. It was found that this self-assembly coating was safe and efficient in promoting re-endothelialization and intimal healing after stent implantation, in addition to having a good hemocompatibility [15]. The anticoagulation effect of heparin has been well recognized. However, extensive use of heparin may lead to bleeding and thrombocytopenia [16]. With this in mind, some researchers have turned to other anticoagulant molecules for developing blood compatible membranes, such as nattokinase and lumbrokinase [17].

Quercetin, a naturally occurring flavonoid, has also been shown to have effects of antithrombosis and anticoagulation similar to heparin. BijakM et al. reported that quercetin had an inhibitory effect on active factor X (FXa) activity, and that it might be a potential structural base for the design of new nature-based, safe, orally bioavailable, direct FXa inhibitors [18]. The coagulation assays conducted by Choi $\mathrm{J} \mathrm{H}$ et al. also demonstrated that quercetin inhibits the enzymatic activity of thrombin and FXa, and suppresses fibrin clot formation and blood clotting [19]. Actually, the antithrombotic effects of quercetin, including the inhibition of the platelet activation and aggregation, in vivo thrombosis inhibition, and human interventional studies on cardiovascular diseases, have been widely reported [20-24]. Furthermore, quercetin has other pharmacological functions, such as antioxidation [25], anti-inflammation [26], and the inhibition of smooth muscle cell proliferation and migration [27]. Therefore, quercetin is also a promising candidate agent for the modification of vascular stents.

Quercetin has a poor aqueous solubility and is easily degradable, which limits its application in the pharmaceutical field. To solve this problem, some studies have focused on the preparation of chitosan nanoparticles loaded with quercetin. Chitosan is a natural, cationic polysaccharide. It is commonly used in drug delivery systems because of its biocompatibility and low toxicity [28,29]. It also plays a critical role in the attachment and growth of endothelial cells [15]. Encapsulation of quercetin into chitosan-based nanoparticles has been reported to efficiently overcome the shortcomings 
of quercetin and to improve its efficiency. Chitosan-based delivery systems are effective in controlling the release of quercetin, which has paved the way for a better bioavailability of quercetin [30].

In this work, we tried to immobilize quercetin onto titanium (Ti) surfaces so as to enhance blood compatibility. We encapsulated quercetin into chitosan nanoparticles based on the ionic interactions between chitosan and tripolyphosphate (TPP). TPP is a polyanion with negatively charged groups that can interact with the positively charged amino groups of chitosan. This ionic gelation process is convenient and mild, and thus it has attracted considerable attention as a way to prepare chitosan nanoparticles [30,31]. The surface charge of quercetin-loaded TPP-chitosan nanoparticles (QTCs) is positive because of the presence of amine groups on its surface [31,32]. Negatively charged hyaluronic acid (HA) and positively charged QTCs were deposited onto a Ti surface and formed a multilayer coating through the LbL technique. Finally, the hemocompatibility of these HA/QTCs multilayers was assessed by platelet-rich plasma contacting experiments. There have been many previous studies that have focused on the surface modification of implant metal via the LbL self-assembly technique [12-15], but, as far as we know, this is the first report of improving the blood compatibility of biomaterials by the surface immobilization of quercetin-loaded multilayer coatings. The results of this study will provide a design reference for the anticoagulant materials.

\section{Materials and Methods}

\subsection{Preparation and Characterization of $Q T C S$}

TPP-chitosan nanoparticles (TCs) were fabricated based on the ionic interactions between the chitosan and TPP anions. Chitosan was dissolved into a $1 \mathrm{vol} \%$ aqueous acetic acid solution in order to form a polymer solution with a concentration of $1 \mathrm{mg} / \mathrm{mL}$. The $\mathrm{pH}$ of the chitosan solution was adjusted to 5.2 using $1.25 \mathrm{M}$ sodium hydroxide. Under magnetic stirring at room temperature, $0.5 \mathrm{~mL}$ of TPP solution, with a concentration of $2 \mathrm{mg} / \mathrm{mL}$, was dropwise added into $5 \mathrm{~mL}$ of the chitosan solution. The QTCs were formed by dropwise adding $1 \mathrm{~mL}$ of quercetin-ethanol solution, with a concentration of $0.2 \mathrm{mg} / \mathrm{mL}$, into $5 \mathrm{~mL}$ of the chitosan solution before adding the TPP.

The encapsulation efficiency (EE) of quercetin was conducted according to the method described in references $[33,34]$. The encapsulated and non-encapsulated portions of quercetin from the QTC suspensions were separated using a centrifugal device with centrifugation at 10,000 rpm for $30 \mathrm{~min}$. The concentration of quercetin in the supernatant liquid was detected by UV-VIS spectrophotometry at $370 \mathrm{~nm}$. The quercetin encapsulation efficiency was calculated according to the following formula:

$$
E E(\%)=\left(W_{\text {total }}-W_{\text {free }}\right) \times 100 / W_{\text {total }}
$$

where $W_{\text {total }}$ is the total quercetin weight in the QTC suspensions and $W_{\text {free }}$ is the weight of the non-encapsulated quercetin.

The particle size and zeta potential of the QTCs were assessed by a Nanotrac Wave II particle size analyzer (Microtrac INC., Florida, USA). The surface morphologies of the QTCs and TCs were observed by transmission electron microscope (TEM; JEOL-2100F, Tokyo, Japan). The UV absorption spectra of the native quercetin, chitosan, and QTCs were obtained in a UV-VIS spectrophotometer (UV-6000PC, Shanghai Metash Instruments Co., Ltd., Shanghai, China).

\subsection{Substrate Preparation}

Commercially pure titanium sheets (From Qinghe Hengzhong Metal Materials Co. Ltd., Xingtai, China) were used as the substrates for the HA/TC and HA/QTC multilayer films syntheses. The Ti plates were mechanically polished to a mirror-like status, then, were ultrasonically cleaned in acetone and ethanol, and finally in deionized (DI) water. The cleaning procedure lasted for $10 \mathrm{~min}$ in each fluid. The cleaned samples were activated in $1.25 \mathrm{M}$ of $\mathrm{NaOH}$ solution at $80^{\circ} \mathrm{C}$ for $24 \mathrm{~h}$, and then thoroughly rinsed with DI water and blown dry. The $\mathrm{NaOH}$-treated Ti samples are referred to from here on as $\mathrm{TiOH}$. 


\subsection{Multilayer Coating Preparation}

The polyethylenimine (PEI) solution was prepared at a concentration of $5 \mathrm{mg} / \mathrm{mL}$ by dissolving PEI (50 wt.\% water solution, $\mathrm{MW} 70,000$ ) in $0.14 \mathrm{M} \mathrm{NaCl}$, and its $\mathrm{pH}$ was adjusted to 4.5 using diluted $\mathrm{HCl}$. The $\mathrm{TiOH}$ substrates were immersed in the PEI solution for $20 \mathrm{~min}$, thus obtaining a precursor layer with a stable positive charge to initiate the LbL self-assembly process. Figure 1 shows an illustration of the construction of the multilayer surfaces. The substrates were first immersed into the anionic HA solution $(1 \mathrm{mg} / \mathrm{mL}$ in DI water, $\mathrm{pH}=5.2$ ) for $15 \mathrm{~min}$ (Step 1), and then rinsed with distilled water and dried with a nitrogen flow (Step 2). Afterwards, the substrates were immersed into the QTC or TC suspensions for $15 \mathrm{~min}$ (Step 3), and subsequently rinsed with distilled water and dried again with a nitrogen flow (Step 4). The $\mathrm{pH}$ of the rinsing water was 5.2. The above steps were repeated $n$ times ( $n=5$ and 10) in order to obtain the films that consisted of n-HA/QTC or -HA/TC bilayers with the QTC or TC coatings on the top.

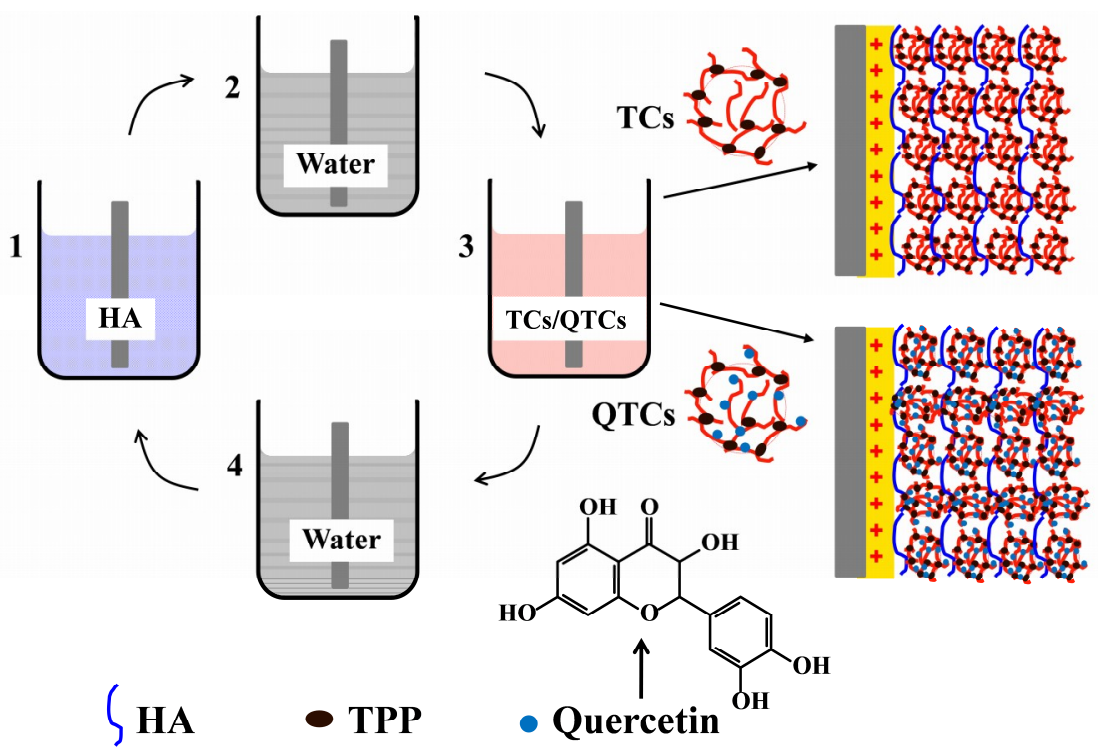

Figure 1. Schematic representation of the construction of hyaluronan acid (HA)/quercetin-loaded tripolyphosphate (TPP)-chitosan nanoparticle (QTCs) and HA/TPP-chitosan nanoparticle (TCs) multilayer thin films by layer-by-layer (LbL).

\subsection{Multilayer Coating Characterization}

The water contact angle of the $\mathrm{TiOH}, \mathrm{TiOH}$ coated with the PEI pre-layer, and HA/QTC films after each alternating deposition were measured to monitor the LbL self-assembly process. All of the prepared coatings were dried with a nitrogen flow before performing the contact angle measurements. The static water contact angles were measured at room temperature using a video contact angle goniometer (SL2008 Powereach, Xiamen, China). The volume of the individual water droplet was $6 \mu \mathrm{L}$, and the contact angle of each type of surface was obtained by averaging the three measurement results performed on the different regions.

To follow the multilayer coating assembly, Ti plates coated with 5 and $10 \mathrm{HA} / \mathrm{QTC}$ bilayers were ultrasonically treated in $1 \mathrm{~mL}$ of anhydrous ethanol for $30 \mathrm{~min}$, separately, after which ethanol solutions containing different concentrations of quercetin were obtained. A UV-VIS spectrophotometer was used to detect the absorption in the UV region of these ethanol solutions.

\subsection{In Vitro Platelet Adhesion Experiments}

In vitro platelet adhesion tests were conducted to evaluate the blood compatibility of all of the samples, including the glass, TiOH substrate, 5 and 10 (HA/TC) bilayer-coated TiOH substrates, and 5 and 10 (HA/QTC) bilayer-coated $\mathrm{TiOH}$ substrates. The entire blood of a healthy rabbit mixed with 
$3.2 \%$ sodium citrate (9:1) was centrifuged at $1000 \mathrm{rpm}$ for $20 \mathrm{~min}$ in order to obtain a platelet-rich plasma (PRP). Each sample $\left(1 \times 1 \mathrm{~cm}^{2}\right)$ was immersed in $1.5 \mathrm{~mL} \mathrm{PRP} \mathrm{and} \mathrm{incubated} \mathrm{in} \mathrm{a} 37^{\circ} \mathrm{C}$ bath for $1 \mathrm{~h}$. After rinsing three times with phosphate-buffered saline (PBS), the samples were fixed with a $4 \%$ paraformaldehyde solution for $1 \mathrm{~h}$ and then dehydrated in graded ethanol $(30 \%, 50 \%, 70 \%, 90 \%$, and $100 \%$; 15 min each) sequentially, and critical point dried with $\mathrm{CO}_{2}$. The quantity, morphology, aggregation, and pseudopodium of the adherent platelets were observed using a scanning electron microscope (SEM; VEGA3 LMH, Tescan, Brno, Czech Republic). Five random regions on each sample were chosen for the statistical analysis.

\section{Results and Discussion}

When the quercetin ethanol solution was dropwise added into the chitosan solution, a transparent light-yellow liquid formed, as shown in Figure 2a. Then, after the incorporation of the TPP solution, the liquid switched to a translucent state with a weak opalescence, and the yellowish color seemed to disappear, as shown in Figure 2b. The process of QTC formation was as follows: The quercetin first adhered to the chitosan based on the electrostatic attraction between the acidic phenolic $\mathrm{OH}$ groups of quercetin and the positively charged chitosan, and then formed nanospheres through TPP-CHI crosslinking under continuous stirring conditions [31,35]. The possible structure of the QTCs is shown in Figure 1. The encapsulation efficiency of quercetin was $91.1 \% \pm 1.2 \%$, indicating that most of the quercetin had been encapsulated in the nanoparticles, and therefore the yellowish color of the solution visually disappeared.

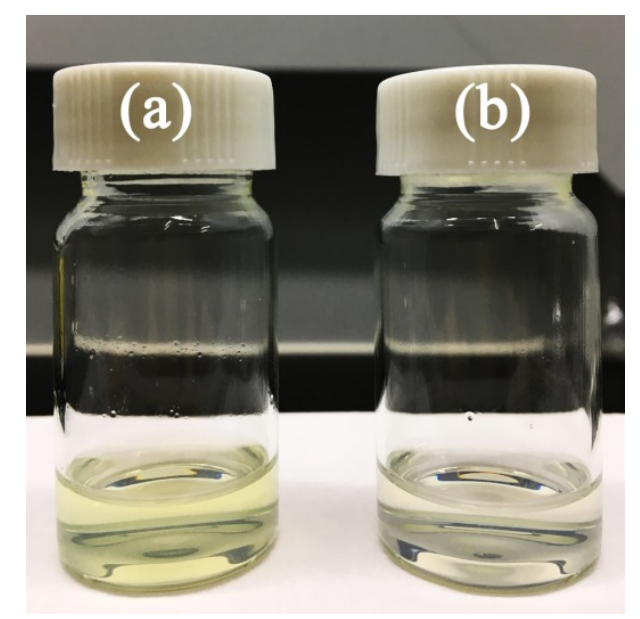

Figure 2. Visual observation of the quercetin-chitosan solution (a) before and (b) after adding the TPP solution.

The size and polydispersity index (PDI) are essential analyses for the characterization of nanoparticles. The dynamic light scattering (DLS) assay showed that the average size of the QTCs was about $279 \mathrm{~nm}$, while the average TC size was $221 \mathrm{~nm}$ (slightly smaller than that of QTCs), as shown in Figure 3a. The polydispersity index of both the quercetin-loaded nanoparticles and the blank nanoparticles was less than 0.25 , which featured a reasonably homogeneous suspension. Figure $3 \mathrm{~b}, \mathrm{c}$ shows the TEM images of the typical morphology of TCs and QTCs, respectively. The nanoparticles were nearly spherical in shape, with sizes ranging from 86 to $160 \mathrm{~nm}$. The average particle size decreased when compared with the results of the DLS analysis, as the nanoparticles had shrunk during the drying procedure prior to the TEM observations. The Zeta potential is a measurement of the surface potential of suspended particles, which is an essential index in the stability of nanoparticles in suspension through the electrostatic repulsion among them. Both the TCs and QTCs had almost the same zeta potential, which was about $45 \mathrm{mV}$ on average. This value is suitable for forming a 
stable nanoparticle suspension, as particles can be dispersed stably when the absolute value of the zeta potential is above $30 \mathrm{mV}[36]$.
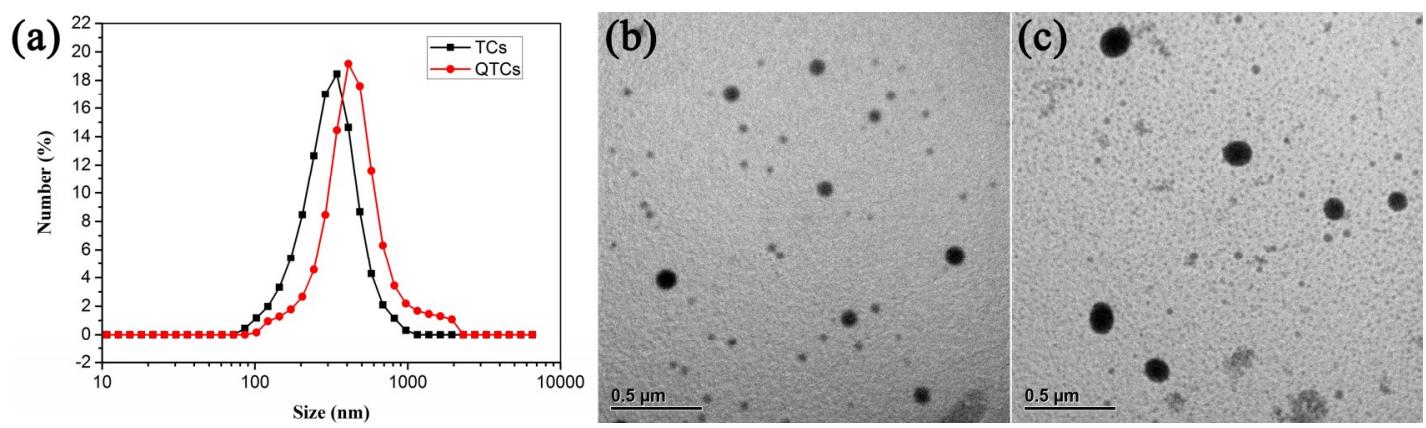

Figure 3. Characterization of TCs and QTCs. (a) Particle size distribution of TCs and QTCs measured by dynamic light scattering, transmission electron microscope (TEM) images of (b) TCs and (c) QTCs.

The UV spectra of the chitosan, QTCs, and native quercetin are presented in Figure 4 (curves a, $\mathrm{b}$, and c, respectively). The native quercetin (dissolved in ethanol) showed two absorption bands at 255 and $370 \mathrm{~nm}$ (curve c), related to conjugations in the B-ring and A-ring, respectively. No apparent absorption peak in the chitosan solution was observed (curve a). In comparison, the spectrum of the QTCs (curve b) showed absorption bands at 255 and $370 \mathrm{~nm}$, indicating the presence of quercetin in the nanoparticles. These results were similar to those of other studies on the encapsulation of quercetin into chitosan nanoparticles [33,34]. The HA/QTCs multilayer films were eluted from the Ti substrates by anhydrous ethanol under a supersonic effect. The obtained ethanol solutions contained different concentrations of quercetin, depending on the number of HA/QTC bilayers. Curves $d$ and e in Figure 4 show the UV absorption spectra of the ethanol solutions obtained from the HA/QTC films with 5 and 10 bilayers, respectively. Two absorption bands at 255 and $370 \mathrm{~nm}$ were also found in each of the two curves, illustrating a successful incorporation of QTCs into the multilayer films. In addition, an increment of the absorbing intensity at 255 and $370 \mathrm{~nm}$ was seen as the number of bilayers increased from 5 to 10, indicating an increase in the amount of QTCs.

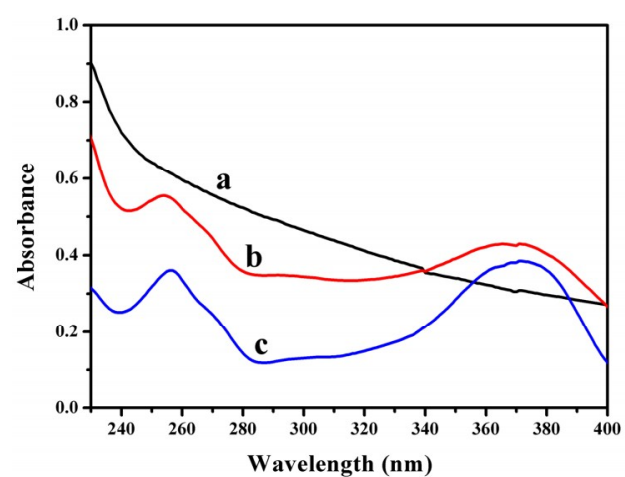

(a)

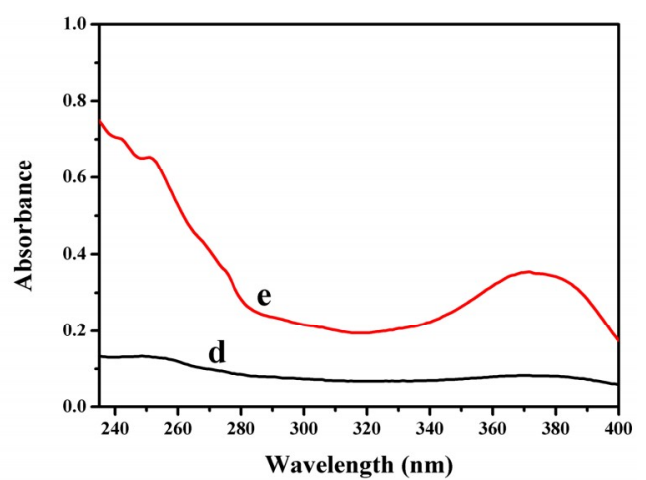

(b)

Figure 4. UV spectra of (a): three types of liquids, a: chitosan solution, b: quercetin-loaded nanoparticles suspension, c: quercetin-ethanol solution, and (b): ethanol solutions obtained from the HA/QTCs multilayer films with d: 5 and e: 10 bilayers.

The wettability of the HA and chitosan coatings had different values; it has been reported that the HA layers are more hydrophilic than the chitosan layers [37,38]. Hence, the LbL assembly of HA/QTCs can be monitored by measuring their respective water contact angles in each of the outermost layer. As seen in Figure 5, the $\mathrm{TiOH}$ sample, i.e., the alkali and heat-treated titanium, had a very hydrophilic surface of $12.1^{\circ} \pm 0.1^{\circ}$. The deposition of the PEI pre-layer on the $\mathrm{TiOH}$ changed its wettability from 
$12.1^{\circ} \pm 0.1^{\circ}$ to $61.8^{\circ} \pm 2.4^{\circ}$. The later repeated coatings of the HA-QTC combinations exhibited a zig-zag effect. The HA layers were more hydrophilic than the QTCs layers, suggesting that alternated HA and QTCs surface layers were successfully achieved.

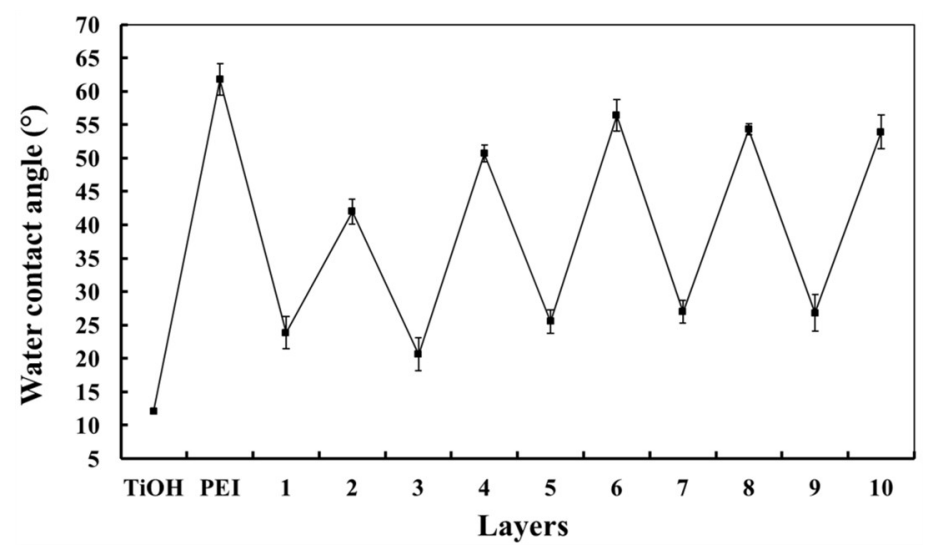

Figure 5. Water contact angles of the HA/QTC multilayer films. The odd numbers on the horizontal axis represent the film with HA as the outermost layer, and the even numbers represent the film with QTCs as the outermost layer.

Platelet adhesion and activation are important markers to evaluate the blood compatibility of blood-contacting materials. Typical SEM images of the adherent platelets on the glass, TiOH substrate, HA/TC-coated TiOH substrates with 5 and 10 bilayers, and HA/QTC-coated TiOH substrates with 5 and 10 bilayers are shown in Figure 6. Many platelets adhered to the smooth surface of the glass plate (Figure 6a). About half of them were round in shape, and the others had developed pseudopodia, which indicated that they had been partially activated. It could be seen that significantly more platelets adhered to the $\mathrm{TiOH}$ substrate (Figure 6b) than to the glass, and most of the adherent platelets developed pseudopodia. Platelet adhesion was significantly reduced in the case of the LbL-modified TiOH substrates with 5 and $10 \mathrm{HA} / \mathrm{TC}$ bilayers (Figure 6c,d, respectively); most of them were isolated. It was easy to conclude that the existence of the HA/TCs LbL coating was efficient in the improvement of the hemocompatibility of the TiOH substrates. However, compared with the glass sample, the platelet adhesion and activation were slightly enhanced on the HA/TC-modified TiOH substrates. Compared with the four previously described substrates, the number of adherent platelets was strongly reduced. There were only a few platelets that could be observed on the surface of the substrate coated with 5 HA/QTCs bilayers, as shown in Figure 6e. Moreover, the platelet adhesion was reduced further when the number of HA/QTC bilayers increased to 10, as shown in Figure 6f. In addition, the platelets on the HA/QTC-modified samples appeared to be slightly smaller than those on the other four substrates, indicating a lower level of activation. These results suggested that the anticoagulation performance of the HA/QTC coatings was very obvious. 


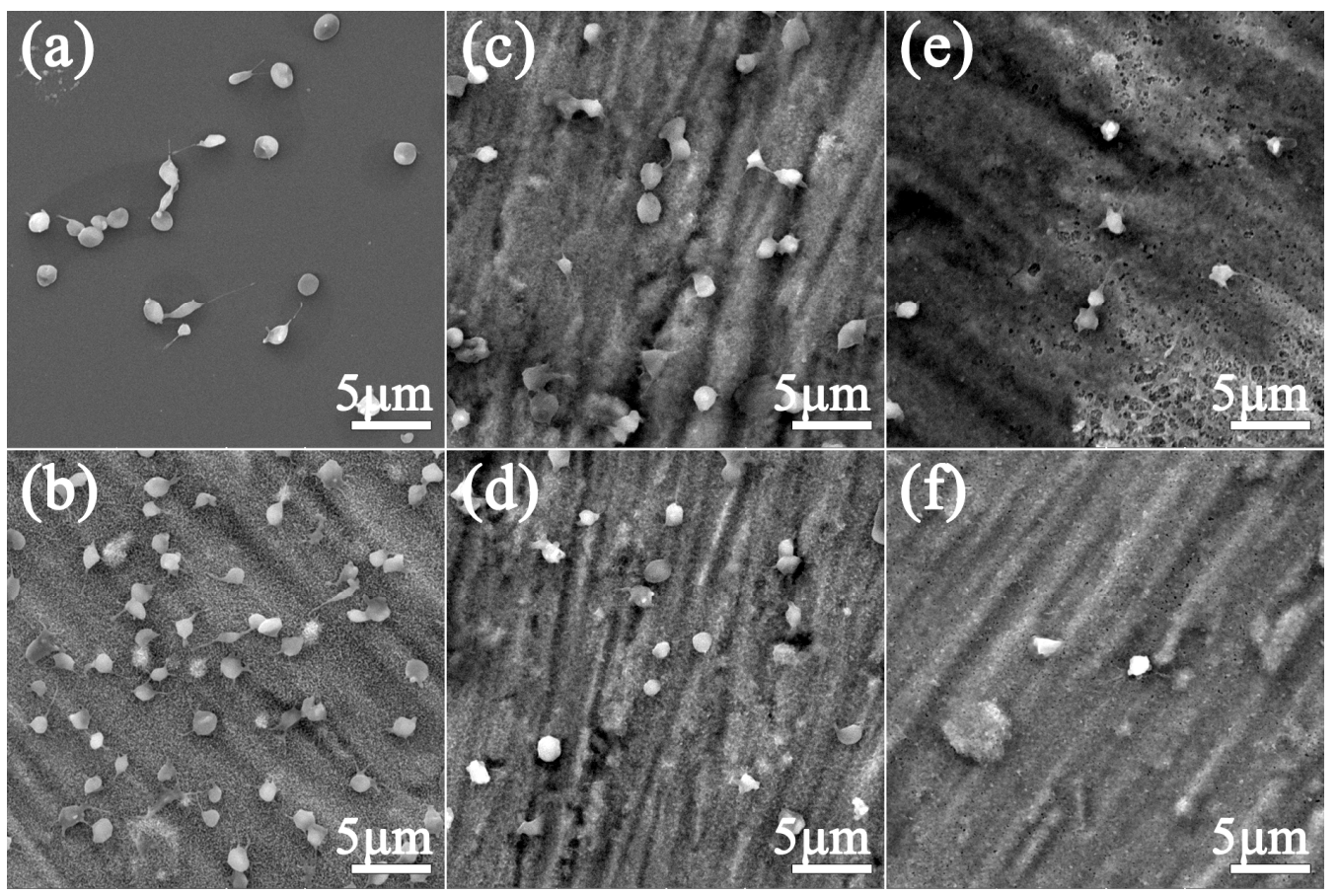

Figure 6. Typical SEM images of platelet adhesion on the surfaces of (a) glass, (b) TiOH substrate, HA/TC-coated Ti substrates with (c) 5 and (d) 10 bilayers, and HA/QTCs-coated Ti substrates with (e) 5 and (f) 10 bilayers.

The statistical number of platelets that adhered to different substrates is shown in Figure 7. The number of platelets that adhered to the surfaces of the glass and the HA/TC-coated TiOH substrates with 5 and 10 bilayers was $15.8 \times 10^{5}, 19.3 \times 10^{5}$, and $17.6 \times 10^{5}$ per $\mathrm{cm}^{2}$, respectively. There was no statistical difference in the number of platelets that adhered to these three substrates. For the $\mathrm{TiOH}$ substrate, the number of adherent platelets was $65.4 \times 10^{5}$ per $\mathrm{cm}^{2}$. While for the substrates coated with 5 and $10 \mathrm{HA} / \mathrm{QTC}$ layers, the numbers of adherent platelets dropped to $1.9 \times 10^{5}$ and $0.2 \times 10^{5}$ per $\mathrm{cm}^{2}$, respectively, which were remarkably less than those on the HA/TC-coated substrates $(p<0.001)$. The number of adherent platelets dropped sharply when the quercetin was incorporated into the modified coatings, which indicated an excellent anticoagulation effect of the quercetin.

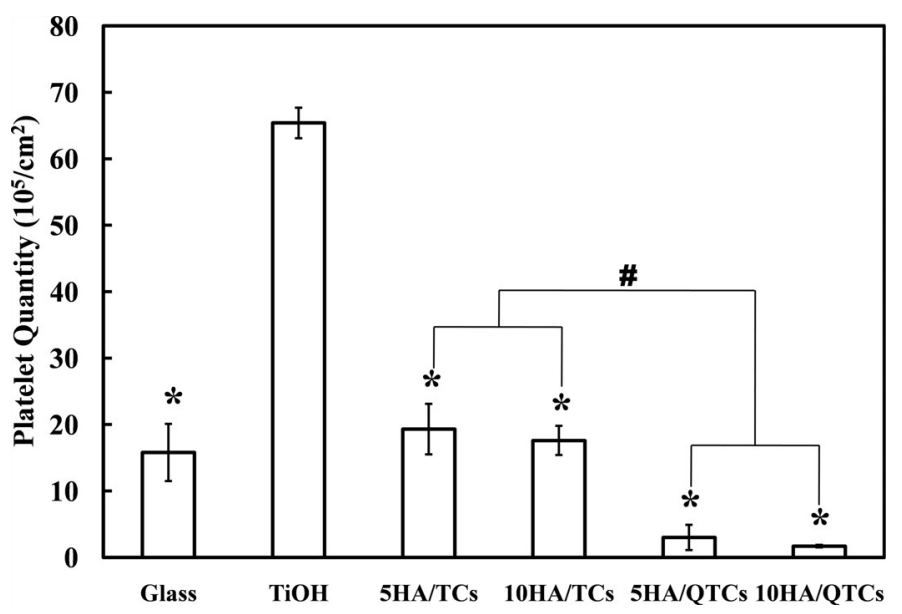

Figure 7. Quantitative analysis of the platelets adhered on the different substrates. * indicates a significant difference ( $p<0.001$, one-way ANOVA) between the TiOH sample and the other five samples. \# indicates a significant difference ( $p<0.001$, one-way ANOVA) between the HA/QTCs samples and the HA/TCs samples. 
Chitosan is a weak base with amino groups, and has a pKa value of about 6.5. The drug-loaded chitosan nanoparticles exhibited a $\mathrm{pH}$-sensitive behavior due to the existence of large quantities of amino groups on the chitosan. The drug release could be controlled by a change of $\mathrm{pH}[28,29]$. The QTCs were formed at pH 5.2 and then deposited onto the TiOH substrates. The latter were incubated in platelet-rich plasma with a $\mathrm{pH}$ greater than 7 . In this process, most of the amino groups on the chitosan were deprotonated, and thus significantly weakened the electrostatic interaction between the QTC and HA layers. Meanwhile, the QTCs became unstable, and quickly collapsed and precipitated, which allowed for a rapid release of quercetin.

Surface modification is a popular method used to improve the blood compatibility of biomaterials. A variety of anticoagulant molecules have been used to modify the surface of blood-contacting implants. Heparin, as an important anticoagulant in clinics, was the most popular surface modifier in earlier studies $[4,5,12-15]$. Subsequently, especially in recent years, other anticoagulant molecules were developed for surface modification, such as citric acid [6], hirudin [7], bovine serum albumin [11], nattokinase, and lumbrokinase [17]. However, to the best of our knowledge, there has been no report of improving the blood compatibility of biomaterials by the surface immobilization of quercetin. Quercetin is well known for its beneficial cardiovascular properties. It has been demonstrated to be effective in reducing or inhibiting platelet aggregation and activation in many studies, although the exact mechanism is not fully understood $[20,21,39]$. However, quercetin has a poor aqueous solubility, and cannot be directly immobilized onto the surface of biomaterials. This problem was efficiently solved in this work by encapsulating quercetin into the $\mathrm{pH}$-sensitive chitosan nanoparticles and constructing multilayer coatings based on the LbL self-assembly of HA and QTC. Alkali and heat-treated titanium was chosen as the platform for the construction of the HA/QTC films. Platelet adhesion experiments demonstrated that the platelet adhesion and activation were reduced remarkably on the quercetin-loaded multilayer coatings. This approach has several advantages. Firstly, the amount of quercetin can be adjusted by simply varying the number of multilayers. Secondly, the alkali and heat-treated titanium has a negatively charged surface and a large number of nanoscale pores, and therefore provides a good platform for the LbL films. Thirdly, according to the reports in the literature [15], the LbL chitosan/heparin coating could significantly promote re-endothelialization, so we speculate that QTCs may also play a role in promoting rapid endothelialization, and further research is underway.

\section{Conclusions}

Quercetin-loaded chitosan nanoparticles were fabricated based on the ionic interactions between chitosan and TPP anions. This study showed that QTCs are stable in suspension, with an average size of $279 \mathrm{~nm}$ and a zeta potential of $45 \mathrm{mV}$. Subsequently, the LbL self-assembly of QTCs and HA through electrostatic interaction was carried out, and the HA/QTC multilayer coatings were successfully constructed, which was confirmed by the UV spectra and water contact angle measurements. Platelet adhesion experiments were carried out with platelet-enriched plasma. Compared with glass, for the $\mathrm{TiOH}$ substrate and $\mathrm{HA} / \mathrm{TC}$-coated $\mathrm{TiOH}$ substrate, a marked reduction in platelet adhesion was observed on the surface of the HA/QTC-coated TiOH substrate, indicating an excellent anticoagulation performance for the quercetin-loaded multilayer coatings. This work provides a promising method to improving the anticoagulant and hemocompatibility of blood-contacting materials.

Author Contributions: Conceptualization, X.W. and H.C.; Methodology, X.W., H.C., and N.T.; Validation, C.L., X.W., and H.C.; Investigation, C.L.; Writing (Original Draft Preparation), X.W.; Writing (Review and Editing), C.L. and H.C.; Visualization, Y.Z.; Project Administration, L.L. and N.T. All authors have read and agreed to the published version of the manuscript.

Funding: This research was supported by "Group-type" Special Support Project for Education Talents in Universities (G619080438 and 4SG19045G), the Scientific Research Project of the Education Department of Guangdong Province (2017KTSCX079), Guangdong Medical Science and Research Foundation (A2018279 and A2019493), Science and Research Projects for Traditional Chinese Medicine of Guangdong (20172086 and 20191191), 
Funds for PhD Researchers of Guangdong Medical University (B2017013), and the Research Fund of Guangdong Medical University (GDMUM201813).

Conflicts of Interest: The authors declare no conflict of interest.

\section{References}

1. Lin, Q.; Yan, J.; Qiu, F.; Song, X.; Fu, G.; Jian, J. Heparin/collagen multilayer as a thromboresistant and endothelial favorable coating for intravascular stent. J. Biomed. Mater. Res. A 2011, 96, 132-141. [CrossRef] [PubMed]

2. Rogers, C.; Parikh, S.; Seifert, P.; Edelman, E.R. Endogenous cell seeding: Remnant endothelium after stenting enhances vascular repair. Circulation 1996, 94, 2909-2914. [CrossRef] [PubMed]

3. Kushwaha, M.; Anderson, J.M.; Bosworth, C.A.; Andukuri, A.; Minor, W.P.; Lancaster, J.R.; Anderson, P.G.; Brott, B.C.; Jun, H.-W. A nitric oxide releasing, self assembled peptide amphiphile matrix that mimics native endothelium for coating implantable cardiovascular devices. Biomaterials 2010, 31, 1502-1508. [CrossRef] [PubMed]

4. Yang, Y.; Qi, P.; Wen, F.; Li, X.; Xia, Q.; Maitz, M.F.; Yang, Z.; Shen, R.; Tu, Q.; Huang, N. Mussel-inspired one-step adherent coating rich in amine groups for covalent immobilization of heparin: Hemocompatibility, growth behaviors of vascular cells, and tissue response. ACS Appl. Mater. Interfaces 2014, 6, 14608-14620. [CrossRef]

5. Gao, A.; Liu, F.; Xue, L. Preparation and evaluation of heparin-immobilized poly (lactic acid) (PLA) membrane for hemodialysis. J. Membr. Sci. 2014, 452, 390-399. [CrossRef]

6. Xiang, T.; Wang, R.; Zhao, W.F.; Sun, S.D.; Zhao, C.S. Covalent deposition of zwitterionic polymer and citric acid by click chemistry-enabled layer-by-layer assembly for improving the blood compatibility of polysulfone membrane. Langmuir 2014, 30, 5115-5125. [CrossRef]

7. Li, J.; Liu, F.; Qin, Y.; He, J.; Xiong, Z.; Deng, G.; Li, Q. A novel natural hirudin facilitated anti-clotting polylactide membrane via hydrogen bonding interaction. J. Membr. Sci. 2017, 523, 505-514. [CrossRef]

8. Xue, T.; Peng, B.; Xue, M.; Zhong, X.; Chiu, C.Y.; Yang, S.; Qu, Y.; Ruan, L.; Jiang, S.; Dubin, S.; et al. Integration of molecular and enzymatic catalysts on graphene for biomimetic generation of antithrombotic species. Nat. Commun. 2014, 5, 3200. [CrossRef]

9. Wei, Y.; Ji, Y.; Xiao, L.; Lin, Q.; Xu, J.; Ren, K.; Ji, J. Surface engineering of cardiovascular stent with endothelial cell selectivity for in vivo re-endothelialisation. Biomaterials 2013, 34, 2588-2599. [CrossRef]

10. Su, Y.; Luo, C.; Zhang, Z.; Hermawan, H.; Zhu, D.; Huang, J.; Liang, Y.; Li, G.; Ren, L. Bioinspired surface functionalization of metallic biomaterials. J. Mech. Behav. Biomed. Mater. 2018, 77, 90-105. [CrossRef]

11. Lin, S.; Li, X.; Wang, K.; Shang, T.; Zhou, L.; Zhang, L.; Wang, J.; Huang, N. An albumin biopassive polyallylamin film with improved blood compatibility for metal devices. Polymers 2019, 11, 734. [CrossRef] [PubMed]

12. Li, G.; Yang, P.; Huang, N. Layer-by-layer construction of the heparin/fibronectin coatings on titanium surface: Stability and functionality. Phys. Procedia 2011, 18, 112-121. [CrossRef]

13. Su, L.C.; Chen, Y.H.; Chen, M.C. Dual drug-eluting stents coated with multilayers of hydrophobic heparin and sirolimus. ACS Appl. Mater. Interfaces 2013, 5, 12944-12953. [CrossRef] [PubMed]

14. Chou, C.C.; Zeng, H.J.; Yeh, C.H. Blood compatibility and adhesion of collagen/heparin multilayers coated on two titanium surfaces by a layer-by-layer technique. Thin Solid Film. 2013, 549, 117-122. [CrossRef]

15. Meng, S.; Liu, Z.; Shen, L.; Guo, Z.; Chou, L.L.; Zhong, W.; Du, Q.; Ge, J. The effect of a layer-by-layer chitosan-heparin coating on the endothelialization and coagulation properties of a coronary stent system. Biomaterials 2009, 30, 2276-2283. [CrossRef]

16. Brieger, D.B.; Mak, K.H.; Kottke-Marchant, K.; Topol, E.J. Heparin-induced thrombocytopenia. J. Am. Coll. Gardiol. 1998, 31, 1449-1459. [CrossRef]

17. Ji, M.; Chen, X.; Luo, J.; Wan, Y. Improved blood compatibility of polysulfone membrane by anticoagulant protein immobilization. Colloids Surf. B Biointerfaces 2019, 175, 586-595. [CrossRef]

18. Bijak, M.; Ponczek, M.B.; Nowak, P. Polyphenol compounds belonging to flavonoids inhibit activity of coagulation factor X. Int. J. Biol. Macromol. 2014, 65, 129-135. [CrossRef] 
19. Choi, J.H.; Kim, K.J.; Kim, S. Comparative effect of quercetin and quercetin-3-O- $\beta$-d-glucoside on fibrin polymers, blood clots, and in rodent models. J. Biochem. Mol. Toxicol. 2016, 30, 548-558. [CrossRef]

20. Mosawy, S.; Jackson, D.E.; Woodman, O.L.; Linden, M.D. The flavonols quercetin and $3^{\prime}, 4^{\prime}$-dihydroxyflavonol reduce platelet function and delay thrombus formation in a model of type 1 diabetes. Diab. Vasc. Dis. Res. 2014, 11, 174-181. [CrossRef]

21. Kaneider, N.C.; Mosheimer, B.; Reinisch, N.; Patsch, J.R.; Wiedermann, C.J. Inhibition of thrombin-induced signaling by resveratrol and quercetin: Effects on adenosine nucleotide metabolism in endothelial cells and platelet-neutrophil interactions. Thromb. Res. 2004, 114, 185-194. [CrossRef] [PubMed]

22. Lee, K.H.; Park, E.; Lee, H.J.; Kim, M.O.; Cha, Y.J.; Kim, J.M.; Lee, H.; Shin, M.J. Effects of daily quercetin-rich supplementation on cardiometabolic risks in male smokers. Nutr. Res. Pract. 2011, 5, 28-33. [CrossRef] [PubMed]

23. Egert, S.; Boesch-Saadatmandi, C.; Wolffram, S.; Rimbach, G.; Müller, M.J. Serum lipid and blood pressure responses to quercetin vary in overweight patients by apolipoprotein E genotype. J. Nutr. 2010, 140, $278-284$. [CrossRef] [PubMed]

24. Yu, P.X.; Zhou, Q.J.; Zhu, W.W.; Wu, Y.H.; Wu, L.C.; Lin, X.; Chen, M.H.; Qiu, B.T. Effects of quercetin on LPS-induced disseminated intravascular coagulation (DIC) in rabbits. Thromb. Res. 2013, 131, e270-e273. [CrossRef] [PubMed]

25. Patel, R.V.; Mistry, B.M.; Shinde, S.K.; Syed, R.; Singh, V.; Shin, H.S. Therapeutic potential of quercetin as a cardiovascular agent. Eur. J. Med. Chem. 2018, 155, 889-904. [CrossRef]

26. Kleemann, R.; Verschuren, L.; Morrison, M.; Zadelaar, S.; van Erk, M.J.; Wielinga, P.Y.; Kooistra, T. Anti-inflammatory, anti-proliferative and anti-atherosclerotic effects of quercetin in human in vitro and in vivo models. Atherosclerosis 2011, 218, 44-52. [CrossRef]

27. Alcocer, F.; Whitley, D.; Salazar-Gonzalez, J.F.; Jordan, W.D.; Sellers, M.T.; Eckhoff, D.E.; Suzuki, K.; Macrae, C.; Bland, K.I. Quercetin inhibits human vascular smooth muscle cell proliferation and migration. Surgery 2002, 131, 198-204. [CrossRef]

28. Sivakumar, P.; Priyatharshni, S.; Nagashanmugam, K.B.; Thanigaivelan, A.; Kumar, K. Chitosan capped nanoscale Fe-MIL-88B-NH2 metal-organic framework as drug carrier material for the $\mathrm{pH}$ responsive delivery of doxorubicin. Mater. Res. Express 2017, 4, 085023. [CrossRef]

29. Tiğlı Aydın, R.S.; Pulat, M. 5-fluorouracil encapsulated chitosan nanoparticles for pH-stimulated drug delivery: Evaluation of controlled release kinetics. J. Nanomater. 2012, 2012, 313961. [CrossRef]

30. Zhang, Y.; Yang, Y.; Tang, K.; Hu, X.; Zou, G. Physicochemical characterization and antioxidant activity of quercetin-loaded chitosan nanoparticles. J. Appl. Polym. Sci. 2008, 107, 891-897. [CrossRef]

31. Nan, W.; Ding, L.; Chen, H.; Khan, F.U.; Yu, L.; Sui, X.; Shi, X. Topical use of quercetin-loaded chitosan nanoparticles against ultraviolet B radiation. Front. Pharmacol. 2018, 9, 826. [CrossRef] [PubMed]

32. Souza, M.P.; Vaz, A.F.M.; Costa, T.B.; Cerqueira, M.A.; De Castro, C.M.M.B.; Vicente, A.A.; Carneiro-da-Cunha, M.G. Construction of a biocompatible and antioxidant multilayer coating by layer-by-layer assembly of $\mathrm{k}$-carrageenan and quercetin nanoparticles. Food Bioprocess Technol. 2018, 11, 1-11. [CrossRef]

33. Hao, J.; Guo, B.; Yu, S.; Zhang, W.; Zhang, D.; Wang, J.; Wang, Y. Encapsulation of the flavonoid quercetin with chitosan-coated nanoliposomes. LWT-Food Sci. Technol. 2017, 85, 37-44.

34. Souza, M.P.; Vaz, A.F.M.; Correia, M.T.S.; Cerqueira, M.A.; Vicente, A.A.; Carneiro-da-Cunha, M.G. Quercetin-loaded lecithin/chitosan nanoparticles for functional food applications. Food Bioprocess Technol. 2014, 7, 1149-1159. [CrossRef]

35. Tan, Q.; Liu, W.; Guo, C.; Zhai, G. Preparation and evaluation of quercetin-loaded lecithin-chitosan nanoparticles for topical delivery. Int. J. Nanomed. 2011, 6, 1621.

36. Liu, J.; Gong, T.; Wang, C.; Zhong, Z.; Zhang, Z. Solid lipid nanoparticles loaded with insulin by sodium cholate-phosphatidylcholine-based mixed micelles: Preparation and characterization. Int. J. Pharm. 2007, 340, 153-162. [CrossRef]

37. Hernández-Montelongo, J.; Nascimento, V.F.; Murillo, D.; Taketa, T.B.; Sahoo, P.; de Souza, A.A.; Beppu, M.M.; Cotta, M.A. Nanofilms of hyaluronan/chitosan assembled layer-by-layer: An antibacterial surface for Xylella fastidiosa. Carbohydr. Polym. 2016, 136, 1-11. [CrossRef] 
38. Nascimento, V.; Franca, C.; Hernández-Montelongo, J.; Machado, D.; Lancellotti, M.; Cotta, M.; Landers, R.; Beppu, M. Influence of $\mathrm{pH}$ and ionic strength on the antibacterial effect of hyaluronic acid/chitosan films assembled layer-by-layer. Eur. Polym. J. 2018, 109, 198-205. [CrossRef]

39. Mosawy, S. Effect of the flavonol quercetin on human platelet function: A review. Food Public Health 2015, $5,1-9$.

(C) 2020 by the authors. Licensee MDPI, Basel, Switzerland. This article is an open access article distributed under the terms and conditions of the Creative Commons Attribution (CC BY) license (http://creativecommons.org/licenses/by/4.0/). 